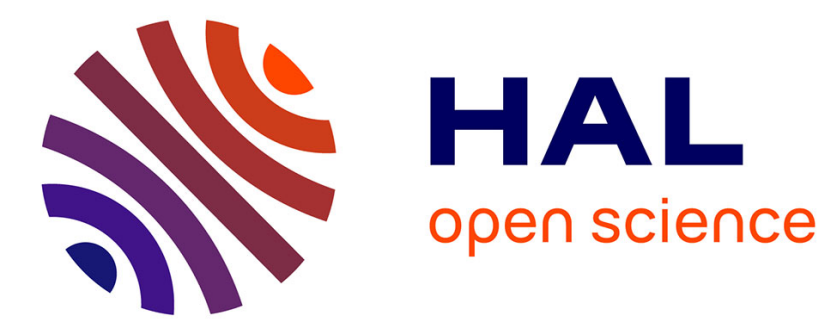

\title{
Les Bretons à La Rochelle d'après le serment au roi de France de 1224 \\ Patrice Marquand
}

\section{To cite this version:}

Patrice Marquand. Les Bretons à La Rochelle d'après le serment au roi de France de 1224. 2011. halshs-00613166

\section{HAL Id: halshs-00613166 \\ https://shs.hal.science/halshs-00613166}

Preprint submitted on 3 Aug 2011

HAL is a multi-disciplinary open access archive for the deposit and dissemination of scientific research documents, whether they are published or not. The documents may come from teaching and research institutions in France or abroad, or from public or private research centers.
L'archive ouverte pluridisciplinaire HAL, est destinée au dépôt et à la diffusion de documents scientifiques de niveau recherche, publiés ou non, émanant des établissements d'enseignement et de recherche français ou étrangers, des laboratoires publics ou privés. 


\author{
Patrice Marquand \\ Université Européenne de Bretagne, F-35000 Rennes, France \\ Université Rennes 2, CRBC, EA 4451, F-35000 Rennes, France
}

\title{
Les Bretons à La Rochelle d'après le serment au roi de France de 1224
}

\section{Introduction : rappel historiographique}

En 1224, dans le cadre du conflit entre Plantagenêts et Capétiens, les bourgeois de La Rochelle ouvrent les portes de leur ville au roi de France Louis VIII et lui prêtent serment de fidélité. Cette prestation de serment fut mise par écrit dans un acte publié dans les Archives historiques du Poitou en 1889'1, d'après une transcription d'A. Bardonnet. L'acte comporte une longue liste de noms qui permet de se faire une idée assez juste de la population rochelaise au début du $13^{\text {ème }}$ siècle, du rayonnement de la ville et de l'étendue de ses relations commerciales.

La première véritable analyse de cette liste fut l'œuvre de Marcel Delafosse en 1955, dans un court article publié dans les Mélanges offerts à Clovis Brunel ${ }^{2}$. L'approche de Marcel Delafosse est anthroponymique, alors qu'elle est démographique et économique pour Yves Renouard, en 1961 dans un article qui fait toujours référence ${ }^{3}$. Yves Renouard est aussi le premier à proposer un décompte des signataires du serment en fonction de leur origine géographique, et à en tirer des conclusions quant au rayonnement économique de La Rochelle au début du 13 ème siècle. Cette liste comprend environ 1750 noms qui correspondent à au moins 1360 personnes différentes. Dans près de la moitié des cas (567, soit plus de $40 \%$ ), le nom est suivi de l'indication du lieu d'origine de celui qui le porte. D'après les calculs d'Yves Renouard, 177 personnes au moins sont originaires d'un lieu distant de plus de cent kilomères de La Rochelle, ce qui représente un tiers des individus dont on connaît l'origine géographique, et treize pour cent de l'ensemble de ceux ayant prêté serment, ce qui est considérable.

Parmi ces étrangers venus s'installer à La Rochelle au début du $13^{\text {ème }}$ siècle, voire quelques décennies auparavant, les Bretons forment le groupe ethnique le plus nombreux. Marcel Delafosse avait comptabilisé 23 Brito, ethnonyme qui renvoie à la Bretagne, Yves Renouard compte quant à lui 27 Bretons. En 1974, André Chédeville relevait dans cette liste 43 Brito, 23 anthroponymes celtiques et 14 surnoms géographiques et, ne retenant finalement pas la seconde catégorie, comptabilisait 57 individus 4 . Jean-Christophe Cassard, dans son ouvrage Les Bretons et la mer, paru en 1998, suit en tous points l'étude de Yves Renouard et ne suggère que quelques rectifications mineures quant à l'origine exacte de deux Bretons ${ }^{5}$.

Le but de notre article est de reprendre complètement cette comptabilité en proposant un décompte détaillé de ces Bretons rochelais par catégories et en pointant les erreurs et oublis commis par les uns et les autres. Afin de situer cette liste dans le contexte politique et

\footnotetext{
1 «Le serment de fidélité des habitants de La Rochelle au roi de France en 1224 », Archives historiques du Poitou, 20, 1889, 232-261.

2 Delafosse, 1955, 339-344.

${ }^{3}$ Renouard, 1961, 79-94.

${ }^{4}$ Chédeville, 1974, 311.

${ }^{5}$ Cassard, 1998, 160-161.
} 
économique de l'époque, la première partie de notre étude résume les conditions dans lesquelles fut créée et se développa la ville portuaire de La Rochelle, alors que la dernière partie avance quelques explications possibles sur la présence des Bretons à La Rochelle en 1224 et dans les décennies qui ont précédé la signature du serment.

\section{1 - Les origines et l'essor de La Rochelle}

$\mathrm{Au} 11^{\mathrm{ème}}$ siècle, en Aunis, les seigneurs de Châtelaillon règnent sur un patrimoine foncier considérable: églises, châteaux, terres sur le continent et dans les îles, vignes, pêcheries et surtout marais salants, la plus importante industrie du secteur, dont ils tirent l'essentiel de leurs revenus. Le sel est pour eux un objet de spéculation : ils l'empruntent lorsqu'il est cher et le rendent lorsque son cours est au plus bas, dégageant ainsi d'importants bénéfices. La ville de Châtelaillon, extrêmement fortifiée, abritait deux ports, le Grand Port et Port Punay, où se concentrait le commerce de l'Aunis, basé principalement sur l'exportation de sel et de vin.

La domination des seigneurs de Châtelaillon s'étend alors sur tout le littoral de la Sèvre à la Charente, sur les îles de Ré et d'Aix et sur une partie de celle d'Oléron. Leur ambition et leur pouvoir se trouvèrent en butte aux intérêts de plusieurs grands monastères propriétaires de terres et de salines en Aunis et à Oléron, ils entrent ainsi en conflit avec les abbayes de Vendôme, de Saint-Maixent et de Saint-Jean d'Angély. Leur politique hégémonique porte aussi ombrage à la volonté de leur suzerain, le duc d'Aquitaine et comte de Poitou, de contrôler l'accès à l'Océan pour ses ports fluviaux du Poitou. En 1130, le duc Guillaume X met le siège devant le château de Châtelaillon et s'en empare l'année suivante ainsi que de tous les domaines de son vassal. La chute des seigneurs de Châtelaillon va permettre l'éclosion et l'essor de La Rochelle'.

En effet, six ans plus tard, en 1137, Guillaume X créé son propre port, un peu plus au Nord : La Rochelle. Désireux de développer rapidement son port personnel, il y attire par une charte de peuplement et des franchises une multitude d'hommes, autochtones ou venant de divers pays et qui affluent tant par terre que par mer. L'activité de la ville nouvelle est centrée autour de son port qui devient vite trop petit. Aliénor d'Aquitaine en fit creuser un second, plus adapté au grand commerce.

Cela explique peut-être qu'Al-Idrîsî ne voit en La Rochelle, avant 1154, qu'une petite ville du Poitou située en bord de mer. Le chroniqueur local Richard le Poitevin, qui rédige vers 1180, décrit au contraire un port adapté à la navigation maritime où une multitude de navires arrive chaque jour de divers pays pour y faire commerce ${ }^{7}$. En effet, les franchises dont bénéficie La Rochelle de la part des ducs d'Aquitaine lui confèrent de grands avantages pour les transactions commerciales dans toute la France de l'Ouest, mais aussi en GrandeBretagne et en Irlande, dès lors que l'Aquitaine sera entrée dans l'espace Plantagenêt.

En 1154, lorsque Henri II Plantagenêt, duc de Normandie et comte d'Anjou, nouveau duc d'Aquitaine par son mariage avec Aliénor, devient roi d'Angleterre, il se constitue, de l'Irlande aux Pyrénées, un nouvel espace politique bordé par l'Océan atlantique. Cet espace

\footnotetext{
${ }^{6}$ Bruhat, 1901 ; Boissonnade, 1924, 276-278 ; Garaud, 1964, 57-60, 145 ; Favreau, 1990.

7 Boissonnade, 1924, 279-281 ; Favreau, 1990, 35-38 ; Al-Idrîsî, V,2, 362.
} 
va favoriser les échanges Nord-sud et principalement le commerce des vins d'Aquitaine vers les Iles britanniques, la Flandre et les Pays-Bas. Il existe alors deux catégories de vins nettement différenciés provenant de deux vignobles concurrents: les vins d'Aunis et de Poitou, exportés par La Rochelle, et les vins de Gascogne exportés par Bordeaux ${ }^{8}$.

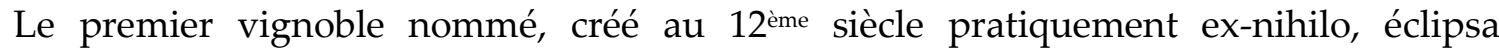
rapidement le second et se tailla d'importantes parts de marché au détriment des vins de Bourgogne et de Loire. Il bénéficiait de deux avantages déterminants : la création de la ville de La Rochelle qui devint très rapidement le plus grand port de la façade atlantique ; le fait que, depuis plusieurs siècles, les pays septentrionaux venaient se fournir en sel en Aunis et Saintonge. L'arrière-pays rochelais, les environs des Sables-d'Olonne et de Saint-Jean d'Angély se couvrent de vignes, le vin et le sel convergent vers La Rochelle pour être exporté vers les ports anglais, flamands et hollandais9.

Le vin de La Rochelle est l'un des protagonistes de la Bataille des Vins, écrite par Henri d'Andeli vers 1223-1224, et qui nous offre un témoignage de premier ordre sur le commerce atlantique $\mathrm{du}$ vin sous Philippe Auguste, commerce qui touche aussi la Bretagne continentale :

Lors dist li vins de La Rocele [...] je repais trestoute Engletiere, Bretons, Flamens, Normans, Englois et les Irois, Norois et cels de Danemarche : jusques la dure bien ma marche! Je suis des vins li sebelins, j'en aport toz les esterlins..$^{10}$

Le vin dit "du Poitou ", chargé à La Rochelle, est mentionné en Irlande ${ }^{11}$ et bientôt en Flandre et en Ponthieu. Guillaume le Breton décrit «...La Rochelle, toute rougie par Bacchus, fière de son port, tel que nul autre n'est plus fréquemment visité par les flottes, et fière aussi de son vin d'Aunis, qui ne le cède en rien celui de Chypre... ${ }^{12}$ » Les vins de Bordeaux, malgré leur excellente réputation ${ }^{13}$ et la protection relative de Jean sans Terre, ne peuvent lutter face à ce commerce de masse.

Il semble que la volonté politique des ducs d'Aquitaine ait été déterminante dans le succès rapide du port de La Rochelle qui reste, jusqu'en 1224, intimement lié au pouvoir des ducs, qu'il s'agisse d'Henri II Plantagenêt, d'Aliénor ou de leurs fils Richard puis Jean. Pour récompenser La Rochelle de sa fidélité pendant la révolte seigneuriale de 1173-74, Henri II Plantagenêt lui concède une commune, la première en date pour le Centre Ouest. Cette fidélité à la couronne d'Angleterre ne se démentira pas pendant la durée du conflit entre Capétiens et Plantagenêts.

Mais en 1224, abandonnés par ces derniers, les bourgeois rochelais ouvrirent les portes de leur ville au roi de France Louis VIII et lui prêtèrent serment de fidélité ${ }^{14}$. Cet événement va contribuer à l'essor du vignoble gascon. En effet, la résistance des Bordelais aux guerres menées contre la Guyenne, d'abord par le roi de Castille, puis par le roi de France, leur vaut

\footnotetext{
8 Renouard, 1952, 5-18 ; 1959, 221-304 ; Boutoulle, 2000, 275-292.

${ }^{9}$ Dion, 1959, 336-364.

${ }^{10}$ Henri d'Andeli, vol. 1, p. 54, v. 116-122.

${ }^{11}$ Giraud de Barri indique que «Le Poitou prend sur sa grande production pour lui faire parvenir des vins en quantité » : Boivin, 1993, 170.

${ }^{12}$ La Philippide, trad. Guizot, p. 385.

${ }^{13}$ Aimeri Picaud trouve excellent le vin de Bordeaux : «Inde, transito quodam maris brachio et flumine Garona, Burdigalensium tellus ; quae vino obtimo et piscibus fertilis. », édition Record, Fita, chap. vii, p. 40-41.

${ }^{14}$ Favreau, 1987, 9-15.
} 
la reconnaissance et l'estime de leur souverain, ainsi qu'une place plus importante réservée à leurs vins sur la table royale. En 1224, lorsque les habitants de La Rochelle prêtent serment au roi de France et tournent le dos au roi d'Angleterre, les ports britanniques se ferment aussitôt à l'accès des navires rochelais, au bénéfice des Bordelais qui deviennent alors les principaux fournisseurs du marché anglais ${ }^{15}$. La Rochelle est devenue le plus grand port français sur l'Atlantique, mais l'île d'Oléron reste anglaise.

\section{2 - Le serment de fidélité et les Bretons}

Le serment au roi de France a donc fourni 177 noms de personnes originaires d'un lieu distant de plus de cent kilomètres de La Rochelle. Ces lieux d'origine lointaine, reportés sur une carte, dessinent les axes du commerce rochelais : deux axes maritimes dont le principal est orienté vers le Nord, jusqu'en Grande-Bretagne, en Normandie et en Flandre, le second, bien moins important, est dirigé vers l'Espagne, le port de Santander et les villes de Pampelune et Saragosse ; un axe maritime et fluvial, remontant la Loire depuis son embouchure pour atteindre le Bassin parisien et la Bourgogne ${ }^{16}$; enfin, un axe continental suivant la route jusqu'à Cahors. On notera que la Gascogne voisine ne fournit qu'un seul nom, conséquence sans doute d'une concurrence déjà en place avec le port de Bordeaux, sur le même produit phare et les mêmes marchés d'exportation ${ }^{17}$. Les Bretons se situent sur l'axe maritime principal. Voici la liste complète de ces Bretons et le détail de nos calculs :

\section{1 - Méthode de calcul :}

Nous avons d'abord relevé la totalité des noms d'origine bretonne contenus dans la liste du Serment. Il n'est pas facile de les compter précisément, du fait de doublons dans la liste (par exemple, il y a neuf Guillelmus Brito). Mais ces doublons portent essentiellement sur des noms très usités à l'époque, comme Guillelmus ou Gaufridus, qui peuvent très bien être portés par des personnes différentes. Nous avons ensuite réparti ces noms en quatre catégories distinctes :

- Les noms formés d'un prénom, breton ou non, et de l'ethnonyme Brito. Certains de ses noms sont en plus qualifiés par un métier.

- Les noms formés d'un prénom, breton ou non, et qualifiés par un surnom breton ou un métier. Dans cette catégorie, nous avons aussi comptabilisé les prénoms bretons seuls.

- Les noms formés d'un prénom, breton ou non, et par un lieu d'origine situé en Bretagne historique.

- Les noms dont l'origine bretonne est incertaine, mais reste possible. Nous les donnons à titre d'information, mais nous ne les avons pas comptabilisés.

\footnotetext{
${ }^{15}$ Dion, op. cit., 365-373 ; Lachiver, 1988, 88-102 ; Boutoulle, 2000, 292-298.

${ }_{16}$ Une voie terrestre par Parthenay et Chinon, gagnant la Loire à Poitiers, est aussi possible, c'est celle qui est privilégiée par Yves Renouard.

17 Voir Renouard, 1961, 84-91.
} 
Voici nos résultats sous forme de tableau :

\begin{tabular}{|c|c|c|c|}
\hline Prénom + Brito & $\begin{array}{l}\text { Prénom + surnom ou } \\
\text { métier }\end{array}$ & $\begin{array}{l}\text { Prénom } \\
\text { d'origine }\end{array}+$ lieu & Incertains \\
\hline Daniel Brito & $\begin{array}{l}\text { Gralandus, } \\
\text { piscionarius }\end{array}$ & $\begin{array}{l}\text { Herveus de Lambal } \\
\times 2\end{array}$ & Alanus Pantor \\
\hline G. Brito & Guillelmus Lagadec & Herveus de Lanion & Cariotus \\
\hline Gaufridus Brito $\times 4$ & Guinganz & Guillelmus de Leone & $\begin{array}{l}\text { Cariotus, } \\
\text { piscionarius }\end{array}$ \\
\hline $\begin{array}{l}\text { Gaufridus Brito, } \\
\text { tabernarius }\end{array}$ & $\begin{array}{l}\text { Guinganz, } \\
\text { cordubanarius }\end{array}$ & Daniel de Aumaria & Guillelmus Lomec \\
\hline $\begin{array}{l}\text { Gaufridus Brito, } \\
\text { cordubanarius }\end{array}$ & Guinganz Lagadec & Guido de Lomaria & $\begin{array}{l}\text { Guillelmus de } \\
\text { Britoco }\end{array}$ \\
\hline Guido Brito & Guinganz Lippus & $\begin{array}{l}\text { Johannes de } \\
\text { Machecol x } 2\end{array}$ & Guillelmus de Britou \\
\hline Guillelmus Brito $x 8$ & $\begin{array}{l}\text { Guinganz, } \\
\text { panetarius x } 2\end{array}$ & Petrus de Macheco & $\begin{array}{l}\text { Helyas Grossus } \\
\text { Oculus }\end{array}$ \\
\hline $\begin{array}{l}\text { Guillelmus Brito, } \\
\text { barbarius }\end{array}$ & Guinganz Rossel & $\begin{array}{l}\text { Renaudus de } \\
\text { Macheco }\end{array}$ & Ivo li Guerrandier \\
\hline Guinganz Brito & Guinganz Roussel & Renerus de Macheco & \\
\hline Haymo Brito & Guiomardus & $\begin{array}{l}\text { Guillelmus de } \\
\text { Nantes }\end{array}$ & \\
\hline Imardus Brito & Guiomarz, furnarius & $\begin{array}{l}\text { Johannes de } \\
\text { Redon[ibus] }\end{array}$ & \\
\hline Ivo Brito, carnifex & Judicellus, arcuarius & $\begin{array}{l}\text { Michael de Redonis } \\
\times 2\end{array}$ & \\
\hline J. Brito & $\begin{array}{l}\text { Judicellus, } \\
\text { carpentarius }\end{array}$ & $\begin{array}{l}\text { Petrus Baart de } \\
\text { Rohan }\end{array}$ & \\
\hline $\begin{array}{l}\text { Johannes Brito, } \\
\text { piscionarius } \times 2\end{array}$ & Judicellus Sarrant & $\begin{array}{l}\text { Ivonetus de Sancto } \\
\text { Paulo }\end{array}$ & \\
\hline Joinus Brito & P. Roalendus & Judicellus de Vannis & \\
\hline Juail Brito & Riolandus & & \\
\hline Judicellus Brito & $\begin{array}{l}\text { Riolandus, } \\
\text { carpentarius }\end{array}$ & & \\
\hline $\begin{array}{l}\text { Judicellus Brito, } \\
\text { cordubanarius }\end{array}$ & $\begin{array}{l}\text { Rivalendus, } \\
\text { carpentarius }\end{array}$ & & \\
\hline Kario Brito & $\begin{array}{l}\text { Rivalendus, } \\
\text { tabernarius }\end{array}$ & & \\
\hline Mauricius Brito & Rivalan, piscionarius & & \\
\hline \multicolumn{4}{|l|}{ Morvent Brito } \\
\hline \multicolumn{4}{|l|}{ Naamont Brito } \\
\hline \multicolumn{4}{|l|}{ Nicholaus Brito } \\
\hline \multicolumn{4}{|l|}{ Riolandus Brito $\times 3$} \\
\hline \multicolumn{4}{|l|}{ Robertus Brito } \\
\hline Robinus Brito & & & \\
\hline
\end{tabular}




\begin{tabular}{|l|c|c|l|}
\hline Roussellus Brito & & & \\
\hline Salomon Brito $\mathrm{x} 2$ & & & \\
\hline Symon Brito & & & \\
\hline Tangui Brito & & & \\
\hline \multicolumn{1}{|c|}{ Total brut } & Total brut & Total brut & \\
\hline $\mathbf{4 4}$ & $\mathbf{2 1}$ & $\mathbf{1 8}$ & \\
\hline
\end{tabular}

Le total brut des noms d'origine bretonne s'élève à 83 . Il faut maintenant déduire les doublons, et d'abord ceux qui sont évidents. Ainsi, Herveus de Lamballe, Johannes de Machecol et Michael de Redonis ne doivent être comptabilisés qu'une fois. Le total pour cette catégorie est donc de 15 noms.

Pour les deux autres catégories, nous proposerons une fourchette de deux nombres dont le plus petit sera calculé après déduction de tous les doublons possibles, alors qu'on obtiendra le plus grand en ne déduisant que les doublons évidents. Ainsi, dans la seconde catégorie, Guinganz panetarius et Guinganz Rossel/Roussel nous semblent être des doublons évidents, alors que Guinganz et Guinganz cordubanarius est un doublon possible, sans plus. Même chose pour Riolandus et Riolandus carpentarius, comme pour Guiomardus et Guiomarz furnarius. Le total de la seconde catégorie peut donc s'établir dans une fourchette de 16 à 19 noms.

La première catégorie est la plus sujette à variations, car le nombre de doublons y apparaît beaucoup plus important. Un seul est évident, car il porte sur les deux termes du nom et sur le métier : Johannes Brito piscionarius. De plus, les doublons possibles concernent des noms très répandus à cette époque, tels que Guillelmus et Gaufridus. Salomon et Riolandus sont également assez courants en Bretagne. Si l'on déduit l'ensemble de ces doublons possibles, nous obtenons une fourchette assez large de 24 à 43 noms.

Le total net des noms d'origine bretonne est donc compris entre 55 et 77.

Concernant les noms incertains, Alanus est un prénom répandu en Bretagne à cette époque ; Cariotus pourrait dériver de Cario, «cher» (cf. Kario Brito) ; Lomec est un nom à consonance bretonne ; Britoco et Britou sont assez proches de Brito ; Grossus Oculus pourrait être la traduction latine du nom breton Lagadec que l'on trouve par deux fois dans la liste, d'autant plus qu'Helyas est un nom biblique, dont la mode est attestée en Bretagne dès le haut Moyen Âge, et qui a perduré bien au-delà, puisque dans on trouve aussi à La Rochelle Daniel, Salomon, Symon et Judicellus/Judicael. Enfin, Guerrandier rappelle la ville de Guérande. On le voit, il s'agit de présomptions qui ne sont cependant pas suffisamment étayées pour permettre de comptabiliser ces noms.

\section{2 - Ecart avec les calculs de Delafosse et Renouard : tentative d'explication}

Marcel Delafosse indique clairement qu'il a réduit à un seul les neuf Guillelmus Brito, y compris Guillelmus Brito barbarius, dont on pourrait pourtant penser que le surnom sert à le différencier des autres Guillelmus. Delafosse a certainement procédé de même pour les autres Brito, et il arrive à un total de 23 pour cette catégorie. Yves Renouard est encore plus restrictif, avec seulement 19 noms, mais la différence s'explique par quatre oublis (Guido, 
Johannes, Morvent, Naamont). La seconde catégorie a été occultée par Delafosse comme par Renouard, alors que des prénoms typiques tels que Guinganz, Gralandus (v. br. Gradlon), Rivalan..., ainsi qu'un surnom comme Lagadec, témoignent de l'origine bretonne de ceux qui les portent.

La troisième catégorie n'est pas détaillée par Delafosse ; Renouard, pour sa part, y comptabilise huit noms, ce qui lui donne un total de 27. Or, il semble évident que, là aussi, il a oublié plusieurs noms: Herveus de Lanion, Johannes Redonibus et surtout les quatre Bretons de Machecoul, Johannes, Petrus, Renaudus et Renerus. Yves Renouard élimine aussi Petrus Baart de Rohan de la liste des Bretons, arguant du fait qu'il existe un FrontenayRohan-Rohan près de Niort. Mais ce bourg des Deux-Sèvres ne portera le nom de RohanRohan qu'à partir de 1714, après être entré, dès le début du $16^{\text {ème }}$ siècle, dans les possessions de la famille bretonne des Rohan ${ }^{18}$. En 1224, seul le nom de Frontenay est connu et Petrus Baart a donc toutes les chances d'être originaire de la seigneurie de Rohan en Bretagne.

\section{3 - Analyse}

Dans leurs calculs, Marcel Delafosse et Yves Renouard ont largement sous-estimé la présence des Bretons. Pour notre part, nous arrivons à un total compris entre 55 et 77 noms, proche du calcul d'André Chédeville. Selon nos calculs, le total des personnes originaires d'un lieu situé à plus de cent kilomètres de La Rochelle passe donc de 177 à une fourchette de 205 à $227{ }^{19}$, dont environ un tiers - de $27 \%$ à $34 \%$ - sont d'origine bretonne. Parmi les 15 noms indiquant une origine précise, quatre viennent de la seigneurie de Machecoul, très impliquée à partir du 11 ème siècle dans le commerce maritime et fluvial lié à l'exportation du sel de la baie de Bourgneuf. Les seigneurs de Machecoul, sires de Retz, entretiennent aussi des relations étroites, matrimoniales, avec les familles seigneuriales du Poitou voisin ${ }^{20}$.

Huit noms sont originaires de ports ou de villes proches du littoral breton : Guillelmus de Nantes, Judicellus de Vannes, Daniel de Aumaria et Guido de Lomaria, c'est-à-dire de Locmaria-Quimper ${ }^{21}$, Ivonetus de Saint-Paul de Léon et Guillelmus de Léon, Herveus de Lanion, Herveus de Lamballe. Enfin, deux individus, Johannes et Michael, viennent de Rennes (Redonibus), et le dernier de Rohan. Douze Bretons de La Rochelle sur les quinze dont on connaît l'origine précise semblent donc avoir un lien avec le trafic maritime entre la Bretagne et l'Aunis.

De même, les métiers exercés par ces Bretons rochelais semblent se rapporter assez souvent à la mer. Ainsi, seize individus sont qualifiés par un métier, dont trois piscionarius, trois carpentarius (charpentiers de marine ?) et trois cordubanarius (cordonniers de marine ?). On notera que ces trois métiers sont parmi les plus exercés dans la population rochelaise, et que les Bretons forment entre 20 et $25 \%$ de ces trois corps de métier. Peut-être faut-il y voir la trace de l'émigration dans une ville portuaire nouvelle de main-d'œuvre qualifiée dans la construction navale et attirée par des conditions d'installation très favorables. En effet, selon Al-Idrîsî, les ports bretons de Vannes et de Raïs ont disposés de chantiers navals avant La Rochelle $^{22}$. Raïs pourrait désigner un port sur le littoral de la vicaria de Retz, ou bien s'appliquer à tout le littoral dépendant des seigneurs de Retz et de Machecoul, entre la Loire

\footnotetext{
${ }^{18}$ Saint-Marc, 1909, 329-358.

${ }^{19}$ En considérant que le total de 150 noms restant, hors Bretons, est exact.

20 Tonnerre, 1998, 155-164.

${ }^{21}$ Cassard, 1998, 160-161.

${ }^{22}$ En tout cas avant le milieu du 12ème siècle : Al-Idrîsî, VI, 1, 418-419.
} 
et l'extrémité nord de l'île de Bouin, c'est-à-dire une bonne partie de la baie de Bourgneuf et de ses salines. Contrôlant à la fois les voies navigables - Tenu et Achenau - assurant le transport du sel jusqu'à la Loire, ainsi que l'accès au littoral de la baie, les seigneurs de Retz devaient percevoir suffisamment de revenus pour disposer des moyens financiers nécessaires à la construction de chantiers navals et au développement d'une véritable économie portuaire.

Enfin, il est difficile, à partir de ces trois catégories de noms, de déterminer la période $\mathrm{d}$ 'installation de ces émigrés Bretons. On peut sans doute supposer que les noms comportant le lieu d'origine appartiennent aux arrivants les plus récents car ce système anthroponymique a été adopté tardivement en Bretagne ${ }^{23}$. Les ethnonymes Brito et les prénoms d'origine bretonne ont pu se maintenir sur plusieurs générations et peuvent correspondre à une émigration plus ancienne, peut-être dès la création de La Rochelle par Guillaume $X$, car cette ville portuaire offre en effet, dès l'origine, un caractère cosmopolite et son activité basée sur le commerce maritime à longue distance attire sans doute rapidement des immigrants étrangers à la région.

\section{3 - Contacts entre la Bretagne et La Rochelle avant 1224}

Outre le serment au roi de France, d'autres documents témoignent de contacts entre la Bretagne et le port de La Rochelle, même avant 1224 : les brefs de mer, les Rôles d'Oléron, et certains récits de croisade et de pèlerinage décrivant un itinéraire maritime atlantique entre la pointe de Bretagne et La Rochelle.

\section{1 - Les brefs de mer}

Il s'agit d'une sorte d'assurance souscrite par les armateurs de navires auprès du duc de Bretagne ou de puissants seigneurs du littoral, et qui les exempte du droit de bris en cas de naufrage sur les côtes bretonnes. Ce droit de bris s'exerçait de temps immémorial sur tout le littoral atlantique et surtout en Bretagne. Il permettait aux seigneurs du littoral de s'accaparer les débris et la cargaison des navires échoués sur leurs côtes après naufrage. Droit régalien au départ, le droit de bris devient, à l'époque féodale, la propriété des seigneurs laïcs mais aussi ecclésiastiques, qui s'enrichissent grâce à lui.

Il semble que la mise en place du système des brefs découle de conventions entre le duc de Bretagne et les ports maritimes du golfe de Gascogne. Les brefs de mer étaient délivrés dans tous les ports bretons de la côte atlantique, du Léon à la baie de Bourgneuf. Ils étaient émis par le duc de Bretagne mais aussi par les vicomtes de Léon et les sires de Retz. A l'extérieur de la Bretagne, on pouvait se les procurer à Bordeaux, La Rochelle et dans d'autres ports poitevins. La décision de les mettre en place témoigne de la nécessité de réglementer et d'assouplir des usages anciens, perçus comme barbares et immoraux par l'Eglise, mais qui entravaient surtout la bonne marche du commerce maritime entre le Sud et le Nord, un commerce en plein développement.

\footnotetext{
${ }^{23}$ Il faut toutefois différencier la Basse-Bretagne et la Haute-Bretagne, celle-ci ayant adopté plus tôt le système nom + lieu. C'est aussi le cas de la noblesse. Cf. Chédeville, 1992, 9-40.
} 
En effet, il semble que la création des brefs soit directement en rapport avec l'augmentation du trafic du vin ${ }^{24}$. Pour B.-A. Pocquet du Haut-Jussé, la plus ancienne mention des brefs de mer est contenue dans un acte du duc de Bretagne Jean IV au profit de l'abbaye de Bégard, daté de 1379, et qui semble reprendre les termes d'un acte plus ancien établi par la duchesse Constance avec l'accord de son fils Arthur. La présence de l'héritier du duché permet de dater l'acte des années 1190 environ, vers la toute fin du 12ème siècle: Arthur est né en 1187, sa mère mourut en septembre 1201. Voici le passage en question :

Comme religieux homes l'Abbé et le Convent de l'Abbaye Nostre-Dame de Begar de l'Ordre de Citeaux en l'Evesché de Treguier nous ayent autrefois donné à entendre, que comme Constance jadis duchesse de Bretaigne, fille du comte Conan nos ancêtres et de l'assentement et volonté d'Artus son fils [...] donna et octroya et confirma à Dieu et à ladite Abbaye [...] la disme de la rente des Nefs, laquelle rente Semimarc est appelée, en quelques lieux, que ladite rente fust prinse en La Rochelle ou ailleurs, à avoir ausdits Moynes et tenir en pure et perpetuelle ausmosne, franchement et entierement à jamais, et qu'ils estoient et avoient esté en saisine et possession d'avoir eu et receu lad. disme des rentes de nostre Duché, que les Nefs doivent qui prennent les petitz seaulx de nos briefs, appellez semy ou demy Marc, en La Rochelle, et en Bretaigne et ailleurs, c'est assavoir des seaulx ou briefs de vingt et cinq soulz, et par les mains de Receveurs des esmoluments et rentes desd. seaulx ou briefs ou temps passé, et par de tant de temps que n'est memoire du contraire, fors le temps des derraines guerres de Bretaigne, $\ldots{ }^{25}$.

L'abbaye de Bégard réclame donc la dîme des brefs ducaux, que leur avait octroyé la duchesse Constance, et dont le paiement avait été interrompu par les guerres. Si le tarif et les dénominations (briefs) appartiennent au 14ème siècle, il n'en demeure pas moins que le fond de l'acte paraît authentique, et qu'il montre une grande similitude avec d'autres actes de la duchesse.

Dans l'acte attribué à Constance, la seule mention de La Rochelle, seule sans Bordeaux, comme lieu extérieur à la Bretagne où l'on délivre les sceaux, témoigne aussi de l'authenticité et de l'ancienneté de cet acte. En effet, en 1262, le plus ancien rôle des comptes du duché de Bretagne indique la présence d'un receveur des sceaux à La Rochelle (Guehenocus Quatre) et aussi à Bordeaux (Pierre Bellouard, puis Durand Grenier). On notera que le tarif mentionne déjà le terme : sceau de demi-marc, toujours en vigueur un siècle plus $\operatorname{tard}^{26}$. Entre la fin du $12^{\text {ème }}$ siècle et 1262 , la situation politique a fait que Bordeaux a connu un essor économique très rapide intimement lié au commerce du vin vers l'Angleterre, ce qui explique la nécessité pour le duc de Bretagne d'ouvrir un bureau de délivrance de sceaux à Bordeaux, sans doute après 1224, date où La Rochelle devient soumise au roi de France.

A La Rochelle, par contre, l'ouverture d'un tel bureau fut sans doute antérieure et contemporaine de la montée en puissance du port de l'Aunis, vers les années 1180, là encore en rapport avec le commerce du vin. On sait qu'avant 1207, le comte de Bretagne percevait des coutumes à La Rochelle (consuetudines de navibus et mercatoribus), dont le roi Jean sans Terre disposa après cette date en faveur de Guillaume de Chantemerle. Robert Favreau

\footnotetext{
${ }^{24} C^{\prime}$ est pour les vaisseaux transportant du vin que le tarif des brefs est le plus élevé : « d'ung vaisseau qui pourra porter forniture de vin, briefz enterrins ", brefs entiers, c'est-à-dire plein tarif. Par contre, il n'y a pas de bref de sauveté pour le sel car, en cas de naufrage, la cargaison est perdue de toute façon. Cf. Planiol, 1896, 468.

${ }^{25}$ Morice, 1742-46, Preuves, II, 232.

${ }^{26}$ Pocquet du Haut-Jussé, 1946, 53-55, 63-64 .
} 
pense que ces coutumes pourraient se rapporter aux brefs de mer $^{27}$. Il nous semble donc qu'on peut suivre James Shephard lorqu'il propose une date de création des brefs dans les années 1190, peut-être à l'initiative de la duchesse Constance ${ }^{28}$. Cette création est un indicateur de la fréquentation des côtes bretonnes, et particulièrement celles du littoral atlantique, par des navires armés à La Rochelle et dans les ports saintongeais et poitevins d'abord, gascons ensuite.

\section{2 - Les Rôles d'Oléron}

La rédaction des Rôles d'Oléron apparaît intimement liée avec le développement du commerce atlantique, et principalement avec le commerce du vin, produit cité dans plus $\mathrm{d}^{\prime}$ un tiers des articles de la version primitive ${ }^{29}$. Or, ce commerce connaît un véritable essor à partir du derniers tiers du $12^{\text {ème }}$ siècle, en parallèle avec la croissance du port de La Rochelle. La Rochelle est peu citée dans les Rôles, deux fois seulement, alors que Bordeaux apparaît à huit reprises ${ }^{30}$, mais les manuscrits conservés traduisent sans doute une situation postérieure à 1224, année où La Rochelle prête serment au roi de France et tourne le dos aux Plantagenêts. C'est le cas du manuscrit B2 de la version bretonne - nommée ainsi car la majorité des manuscrits de cette version ont été insérés dans la Coutume de Bretagne -, qui omet de mentionner La Rochelle dans l'article 1, alors que le port est cité dans la version primitive ${ }^{31}$.

Par ailleurs, le fait que Bordeaux soit la ville la plus mentionnée dans les Rôles, alors qu'elle est demeurée fidèle à la couronne d'Angleterre, est une preuve de plus que les Rôles d'Oléron ont été rédigés à l'intérieur de l'espace Plantagenêt. Et les deux mentions de La Rochelle montrent aussi que la rédaction primitive des Rôles est probablement antérieure à 1224, à une période où La Rochelle détenait le quasi monopole du commerce vinaire entre l'Aquitaine et les îles britanniques. Les relations commerciales entre La Rochelle et l'Angleterre sont bien attestées à partir des années 1180, où des marchands rochelais sont présents à Londres. De même, des marchands anglais sont signalés en Aunis dès le milieu du $12^{\text {ème }}$ siècle. A la fin du $12^{\text {ème }}$ siècle, l'armateur Nicolas de Gloucester traite avec les Templiers de La Rochelle, et seize bourgeois d'origine anglaise signent le serment de fidélité à Louis VIII en $1224^{32}$.

Si les négociants ont donc du s'adapter aux changements politiques, ce n'est sans doute pas pour autant le pouvoir politique qui leur a imposé la réglementation compilée dans les Rôles. L'introduction du manuscrit de La Haye, le plus récent (1517-1519), a pu donner à

\footnotetext{
${ }^{27}$ Rotuli litterarum patentium, I-1, p. 73b et 77b ; Favreau, 1987, 24.

${ }^{28}$ Shepard, 1983, 120-126.

${ }^{29}$ Dans neuf articles sur vingt-quatre exactement. La version dite primitive comporte vingt-quatre articles et constitue la base des versions dites «bretonne» (26 articles) et du Black Book of Admiralty (35 articles). Voir Shephard, 1983, 66-77; 1985, 12-42.

${ }^{30}$ Articles $n^{\circ} 1$ et 13 pour La Rochelle (dans le second cas, il s'agit d'un ajout sur trois manuscrits), $\mathrm{n}^{\circ} 1,4,8,11,13$, 18, 19 (ajout sur un manuscrit) et 21 pour Bordeaux.

31 Voir Shepard, 1985, p. 96.

32 Shepard, 1983, 102-105. On peut ajouter à ce total un Cornouaillais, un Gallois et deux Irlandais. Pour Yves Renouard, 1961, 91-92, les Anglais de La Rochelle ont signé le serment avant tout pour préserver leurs intérêts économiques.
} 
penser que Richard Cœur-de-Lion était à l'origine des Rôles ${ }^{33}$, d'autres historiens y ont vu l'œuvre d'Aliénor, mais aucun preuve sérieuse n'a pu être apportée pour confirmer ce qui relève plutôt de la tradition populaire ${ }^{34}$. Par contre, il est indéniable que les Plantagenêts ont favorisé au possible l'essor du commerce atlantique, notamment en faveur de La Rochelle, et que la réunion du royaume d'Angleterre, des duchés de Normandie, d'Aquitaine et de Bretagne sous l'autorité du même suzerain a formé un contexte favorable au développement de relations économiques entre ces territoires. Il est donc fortement probable que la version originale des Rôles d'Oléron fut rédigée après 1154, année où Henri II Plantagenêt, déjà comte d'Anjou, duc de Normandie et duc d'Aquitaine par son mariage avec Aliénor, accède au trône d'Angleterre, mais avant 1224, année où La Rochelle sort de l'espace Plantagenêt.

Où fut rédigé la version originale des Rôles d'Oléron ? On sait, par les manuscrits de la version bretonne, qu'un exemplaire des Rôles était utilisé sur l'île en 1286, le nom d'Oléron apparaît en introduction du premier article, et en conclusion des manuscrits de cette version bretonne ${ }^{35}$, mais l'île n'est concernée par aucun des articles. En 1213, Oléron figure cependant parmi les ports fréquentés par les marins bayonnais regroupés en corporation, mais c'est surtout à La Rochelle que ces navires venaient charger leur fret avant de prendre la route de la Flandre ${ }^{36}$.

Les Rôles d'Oléron réglementent les rapports entre les affrêteurs et les capitaines de navires. Ils offrent un cadre juridique au contrat liant le capitaine, responsable du navire, à l'affrêteur, qui apporte la cargaison, constituée le plus souvent de tonneaux de vins. Dès la fin du 12ème siècle, La Rochelle dispose à la fois de la production viticole fournie par son arrière-pays, des négociants-affrêteurs pour la commercialiser, et des navires pour la trasnporter. Après 1224, le port de La Rochelle étant fermé aux bateaux anglais ou alliés de l'Angleterre, Oléron, restée fidèle aux Plantagenêts, prend une importance cruciale dans le commerce du vin, tout comme Bordeaux.

Mais l'île d'Oléron ne produit pas assez de vin pour alimenter le commerce atlantique, tandis que Bordeaux n'a pas les navires et les marins nécessaires pour assurer l'exportation de sa production viticole. En 1212, Jean sans Terre fit venir d'Oléron en Angleterre trente tonneaux de vins ${ }^{37}$, mais rien ne prouve que ce vin fut produit sur place, cela vient simplement renforcer le fait qu'Oléron commence à devenir une place importante dans le réseau commercial atlantique.

Quant à Bordeaux, c'est un port sans armateurs et sans marins, qui abandonne à d'autres le soin de transporter ses vins ${ }^{38}$. D'autre part, les Rôles édictent des règles en évolution constante, si bien qu'il circula très vite plusieurs versions en parallèle. Aussi, il est fort possible qu'une des versions ait été rédigée à Oléron, après 1224, entre marins locaux et

\footnotetext{
${ }^{33}$ Cette introduction présente les Rôles d'Oléron comme étant la « coustume de la mer faicte entre l'empereur de Romme et le roi Richart observez à Bordeaulx, Angleterre, Britaigne, Normandie, Ecosse et en Pruyche. »Cité par Shephard, 1983, 48.

34 Voir les synthèses de James Shephard, 1983, 141-145 et de Michel Deniel, 1971, 133-139.

35 «Est la copie de le carte d'Oleiron des jutgementz de le mer. » (art. 1), «Dont tesmoing le seel de l'ille de Ouleron establi. » (art. 27), cf. Shephard, 1985, p. 53 et 84.

36 « Res quoque et mercandise, que affretabuntur in nauibus que debent nauigari in Ruppelam uel Burdegalam uel Roianum uel Oleronem aut inter duas terras, esse [debent] de societate. ", Constitutio societatis navium Baionensis, éd. Pardessus, 1828, IV, 286-289. La Rochelle est encore citée plusieurs fois dans le texte, en relation avec la Flandre. Voir aussi les commentaires de James Shephard, 1983, 126-128.

${ }^{37}$ Cf. Dion, 1959, 360-361.

38 Voir Bernard, 1968, I, 17-21.
} 
affrêteurs bordelais. Avant cette date, La Rochelle cumulait tous les atouts pour voir naître la rédaction originale.

Dans la version primitive des Rôles, quatre articles traitent des naufrages et des risques de mer, deux portent sur les pilotes. Avec l'essor du commerce atlantique, la probabilité de perdre un navire et sa cargaison dans un naufrage augmente proportionnellement à la croissance du trafic maritime. La connaissance des dangers du littoral, des zones à risque (écueils, bancs de sable, tempêtes) est indispensable à la bonne marche des affaires, il est donc nécessaire de faire appel à des pilotes locaux et chevronnés, et c'est ce que prévoit l'article 13 des Rôles :

Une nef frete à Bourdeux (var. ou à La Rochelle) ou aillors et vient à sa descharge, et font chartre-partie : toatge et petitz lecmans sont sus lez marchanz; en le coste de Bretaingne, touz ceus que l'en prent puis que l'en a passé l'ile de Baz on Leon (var. et Louessant) sont petitz lezmans et ceus de Normendie et de Engleterre puis que l'en a passé Genere et ceus de Flandres puis que l'an [a] passé Calais, et ceus d'Escoce puis que l'en [a] passé Jarnemue. Et ce est le jugement en cest cas.

Un navire frète à Bordeaux (ou à La Rochelle) ou ailleurs, arrive à l'endroit du déchargement, et [le maître et les marchands] font une charte-partie : le touage et le lamanage sont aux frais des marchands ; sur la côte de Bretagne, tous ceux que l'on prend après que le navire ait dépassé l'île de Batz en Léon (et Ouessant) sont des lamaneurs ; [il en est de même] pour ceux de Normandie et d'Angleterre après que l'on ait dépassé Guernesey, et ceux de Flandre après que l'on ait dépassé Calais, et ceux d'Ecosse après que l'on ait dépassé Yarmouth. Et c'est le jugement en ce $\operatorname{cas}^{39}$.

Le Coutumier d'Oléron, rédigé au $14^{\text {ème }}$ siècle, précise que le petit loman ou lamaneur est celui qui loge à l'entrée des ports et des havres et en connaît les dangers ${ }^{40}$. Il s'agit donc d'un pilote indigène embauché sur place, pour une mission bien précise qui est de faire passer les zones côtières dangereuses aux navires qui l'engagent.

Or, on sait par ailleurs qu'à côté du bref de sauveté, qui garantissait à l'armateur la propriété de son épave et de sa cargaison, déduction faite des frais de sauvetage, les vicomtes de Léon mirent en place dans le port de Saint-Mathieu des coutumes propres dont le paiement était obligatoire sous peine de poursuites. La première donnait aux navires un droit d'escale et de ravitaillement dans les ports de la vicomté et leur évitait la saisie en cas de débarquement sans autorisation. Cette coutume avait cours dans les autres ports bretons, et notamment à Pornic. Par contre, il semble que la seconde était spécifique au Léon. Par son acquittement, le capitaine du navire obtenait la mise à disposition d'un pilote du cru, spécialiste de la navigation côtière et de ses pièges, qui l'aidait à franchir les raz et la pointe Saint-Mathieu. Il recevait parfois le renfort d'une escorte assurant sa sécurité en cas de besoin.

Ces coutumes sont citées dès le milieu du $13^{\text {ème }}$ siècle, mais ne sont connues dans le détail que par la Très Ancienne Coutume de Bretagne, au début du 14 ${ }^{\text {ème }}$ siècle. Lorsqu'à la fin du $13^{\text {ème }}$ siècle, les ducs de Bretagne acquirent les droits des vicomtes de Léon, les

\footnotetext{
39 Ed. Shephard, 1985, 67-68 et notes p. 117-118. Nous avons légèrement modifié la traduction en français moderne.

${ }^{40}$ Bémont, 1919, 330, § 168 ; Williston, 1992, 99, 177.
} 
coutumes léonardes se trouvèrent confondues avec les brefs ducaux, qui se divisèrent alors en trois types distincts : brefs de sauveté, brefs de victuailles et brefs de conduite ${ }^{41}$. Ces dispositions accompagnent généralement les Rôles d'Oléron dans les manuscrits bretons. Elles ont été compilées au début du $14^{\text {ème }}$ siècle dans la Très Ancienne Coutume de Bretagne ${ }^{42}$. L'article 13 reprend donc en substance la coutume léonarde concernant la mise à disposition de pilotes indispensables au franchissement sans encombre de la pointe du Finistère.

L'article 8 stipule que si un navire, pris dans une tempête, ne peut y échapper sans jeter à la mer tout ou partie de sa cargaison, vin compris, les marchands ne pourront s'y opposer ${ }^{43}$. Or, un passage de la Vie de saint Maurice de Carnoet semble se référer à cette disposition : un navire venant de faire escale à l'île de Groix et se rendant à Quimperlé, avait du jeter à la mer sa cargaison de vin pour ne pas sombrer dans la tempête. Cet incident est antérieur à 119144. L'article 17, s'il ajoute à la mauvaise réputation des Bretons quant à leur consommation d'alcool, montre d'abord et surtout que des marins des côtes de Bretagne participaient au commerce atlantique du vin, avec leurs confrères normands :

Les marinaus de la coste de Bretainhe ne deivent avoir que une quisine le jour par la rezon que ils ont bevrage alant et venant; et ceus de Normandie en doivent dous le jour par la rezon que le mestre ne lour baille que eyue à l'aler. Mes pesque le nef est en le terre o le vin creist les marinaus en doivent avoir bevrage et lour doit querre le mestre. Et ce [est] le jugement en cest cas.

Les marins de la côte de Bretagne ne doivent avoir qu'un repas par jour parce qu'ils ont du vin à l'aller et au retour ; et ceux de Normandie doivent avoir deux repas par jour parce que le maître ne leur donne que de l'eau à l'aller. Mais lorsque le navire se trouve dans un lieu où il $\mathrm{y}$ a des vignobles, le maître est tenu de se procurer du vin pour ses marins. Et c'est le jugement en ce cas ${ }^{45}$.

La Bretagne n'a pas seulement fourni des marins au commerce maritime, elle semble aussi avoir pris une part active, si ce n'est à la rédaction des Rôles d'Oléron, au moins aux négociations qui ont du précéder celle-ci. En effet, l'essor du commerce maritime a été freiné par le droit de bris qui permet aux pouvoirs littoraux de s'accaparer les épaves et leur cargaison rejetées sur les côtes lors des naufrages. Les seigneurs bretons sont particulièrement attachés à cette coutume qui leur procure des revenus non négligeables.

Or, les articles des Rôles d'Oléron relatifs aux naufrages ne peuvent se comprendre que si ce droit de bris n'a plus cours : il y est question de l'obligation des marins à sauver ce qu'ils peuvent du gréement et de la cargaison, l'ensemble pouvant être mis en gage, voire même vendu par le maître du navire, avec l'accord de l'armateur, afin de payer les marins et leur permettre de rentrer chez eux. Si la cargaison a été sauvée par l'équipage, le marchand ne pourra la récupérer que s'il paie le fret correspondant à la partie du trajet effectuée ${ }^{46}$. Ces

\footnotetext{
${ }^{41}$ Planiol, 1935-55[1981-84], IV, 186-191 ; Touchard, 1956, 116-140 ; Pocquet du Haut-Jussé, 1959, 255-262 ; Darsel, 1966, 1-14.

${ }^{42}$ Editée par Planiol, 1896, 465-468.

${ }^{43}$ Shepard, 1985, 60-62.

${ }^{44}$ Plaine, 1881, 22-23, 49-51 ; Cassard, 1998, 160.

${ }^{45}$ Shepard, 1985, 72-73.

${ }^{46}$ Shepard, 1985, 54-57, art. $3 \& 4$.
} 
dispositions sont incompatibles avec le droit de bris qui transfère la pleine propriété du navire et de sa cargaison au seigneur maître des côtes sur lesquelles le navire s'est échoué. Ces articles n'ont pu être rédigés qu'après abandon du droit de bris par les seigneurs du littoral atlantique, et en particulier par les seigneurs bretons.

En fait, en Bretagne, ce droit n'a pas été supprimé, mais il a été atténué par l'instauration des brefs de mer, qui ne sont rien d'autre qu'une prime d'assurance dont le paiement par 1' affréteur évite que le droit de bris soit appliqué à sa cargaison en cas de naufrage. La création des brefs résulte donc d'une négociation entre d'une part les armateurs, marins et marchands du littoral aquitain, et en premier lieu, sans doute, de La Rochelle, et d'autre part le pouvoir ducal breton et ses vassaux, lesquels ont du subir une certaine pression politique de la part de leur suzerain (en l'occurrence la duchesse Constance), mais probablement aussi du roi d'Angleterre lui-même. Certains articles des Rôles d'Oléron n'ont pu être écrits qu'après la mise en place des brefs de mer, c'est-à-dire après 1190. Cela nous permet de resserrer la fourchette chronologique dans laquelle s'inscrit la rédaction de la version originale des Rôles : nous proposons de la dater entre 1190 et 1224 .

\section{3 - Les itinéraires atlantiques et les pilotes}

En 1189, une dizaine de navires germaniques s'embarque à Brême pour participer à la troisième croisade. Leur périple est conté par un clerc allemand, membre de l'expédition, dans un journal de bord qu'il finalise à son retour de croisade, vers 1191, la Narratio de itinere navali peregrinorum Hierosolymam tendentium et Silviam capentium ${ }^{47}$. Partis de Brême, les navires font escale à Dartmouth (Devonshire) d'où ils repartent le 25 mai 1189 afin de gagner la Terre Sainte, mais ils sont bientôt retardés par des vents contraires et, après six jours en mer et une escale à Saint-Mathieu, c'est la tempête qui les oblige à se dérouter en direction de Belle-île où ils passent huit jours avant que des vents favorables ne les poussent jusqu'à La Rochelle. L'auteur précise que la Bretagne compte neuf évêchés dont trois utilisent la langue bretonne et nous donne en plus une variante locale du nom de Belle-île, Wechele ${ }^{48}$.

Ce texte montre que Saint-Mathieu est une étape obligée entre la mer du Nord et le golfe de Gascogne et que la route habituelle est directe jusqu'à la Péninsule ibérique. La suite du récit nous apprend en effet que les navires allemands, après avoir quitté La Rochelle, gagnent la haute mer et font escale à Gozon. Ils poursuivent ensuite leur périple le long de la côte cantabrique et vont visiter Saint-Jacques de Compostelle. Outre la route directe, il existait aussi une route de cabotage qui longeait la côte, de la Bretagne à l'Aunis. Les navires allemands l'empruntent à partir de Belle-île jusqu'à La Rochelle, décrite comme " une très opulente forteresse ", ce qui confirme le témoignage de Richard le Poitevin vers 1180 et montre l'évolution rapide de la cité, par rapport au récit d'Al-Idrîsî.

\footnotetext{
${ }^{47}$ Ed. C.W. Wendell, 1939, 591-676 ; éd. partielle avec trad. fr. par Jeanne-Rose, 2001, 199-201.

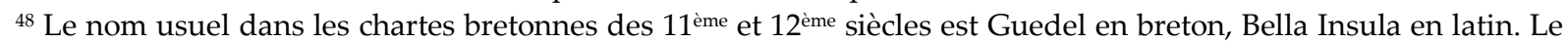
nom antique est Vindilis, d'où dérive Guedel et sans doute aussi Wechele, qui parait être une forme plus conservatrice, l'évolution uu- = w- > gu- en début de mot s'étant produite au cours des $10^{\text {ème-11 ème }}$ siècles. Quant à la mention des trois évêchés utilisant la langue bretonne (il doit s'agir des évêchés de Cornouaille, Léon et Tréguier), nous comprenons que la prédication y est faite exclusivement en breton à destination d'une population totalement monolingue. A cette époque, il y a encore des locuteurs bretons dans les diocèses de Vannes, SaintBrieuc, Alet, Dol et même Nantes (pays de Guérande). Voir les annotations de C.W. David dans son édition de la Narratio, 1939, pp. 612-613, n. 27, 38 et 41.
} 
Ces deux routes sont explicitement décrites dans le De viis maris, texte édité récemment par Patrick Gautier-Dalchét9. Il forme un ensemble avec deux autres textes, probablement du même auteur et présents dans les mêmes manuscrits : l'Expositio mappe mundi, qui est la description d'une mappemonde semblable à celle d'Hereford (fin $13^{\text {ème }}$ siècle), et le Liber Nautarum, qui traite des noms d'objets ou de fonctions relatifs aux navires, des saisons et des vents, en s'inspirant en partie des Etymologies d'Isidore de Séville. Le De viis maris n'est pas un récit de croisade, mais il a pourtant été rédigé par un chroniqueur de premier plan, historien et clerc royal, Roger de Howden, entre août 1191 et le printemps 1193, conjointement avec les Gesta regis Henrici II et Ricardi I et peu avant la Chronica. Les trois œuvres ont d'ailleurs des rapports étroits entre elles, l'auteur ayant utilisé les mêmes renseignements, en les adaptant différemment en fonction du but qu'il poursuivait pour chacune d'elles.

Le De viis maris est un exposé minutieux sur les ports et la physionomie du littoral atlantique qui propose une description précise et détaillée de l'approche de ces ports, des passages particulièrement dangereux (récifs, écueils) à proximité des côtes, ainsi que des instructions sur les vents, les marées et l'état des fonds marins. Il donne aussi bon nombre $\mathrm{d}$ 'indications sur les distances et les temps de navigation entre deux escales. Si le De viis maris se place dans le même ensemble que les récits de croisades maritimes, car décrivant lui aussi les routes maritimes depuis la Manche jusqu'en Palestine et en Egypte, il est beaucoup plus concret et systématique quant aux réalités de la navigation, et se place toujours du point de vue du navire. Pour le rédiger, Roger de Howden a du utiliser un routier de la Manche et de l'Atlantique.

Il s'agit à la fois d'un itinéraire hauturier et d'un itinéraire par cabotage, qui divergent à partir de Saint-Mathieu. Après avoir décrit les côtes anglaises depuis son Yorkshire natal jusqu'aux points d'embarquement habituels des navires croisés (Darmouth, Start Point), Roger de Howden nous fait traverser la mer Britannique en direction de l'île de Batz, puis s'engage dans le chenal du Four où s'échelonnent les bons ports de Munrealis ( ?), Sanctus Mallon - peut-être l'île Melon, au large de Porspoder, plutôt que Saint-Malo, beaucoup plus à l'est -, Le Conquet, et le havre de la pointe Saint-Mathieu. Le De viis maris donne alors de façon très précise la marche à suivre pour aller directement en Espagne : il faut passer entre le rocher du Four et Ouessant, puis entre Saint-Mathieu et l'île Beniguet, et gouverner tout droit jusqu'en Espagne, sans dévier ni à droite, ni à gauche.

Si l'on souhaite se rendre en Poitou, il faut obliquer à gauche pour rejoindre la baie de Douarnenez avec les ports de Poullan et de Pouldavid, puis passer le raz de Sein, île qui dispose aussi d'un port. Le De viis maris avertit du danger que constituent les écueils invisibles à l'entrée de celui-ci et le rocher de Kasa, au milieu du raz (s'agit-il du Pont des Chats ?). L'itinéraire se poursuit par les ports de Penmarc'h, des Glénans, du Blavet, puis par l'estuaire de la Loire où se trouve le port de La Garnache. Les îles de Noirmoutier, d'Yeu et de Belle-île sont citées. Puis, on arrive en Poitou, au port d'Olonne, avant de gagner Le Plomb et La Rochelle et enfin, l'estuaire de la Gironde et Bayonne.

L'itinéraire rédigé par Roger de Howden présente certains points communs avec la route suivie par les navires croisés allemands en 1189: le cabotage commence à SaintMathieu, on retrouve Belle-île et La Rochelle. La nature de la source dont dérive le De viis maris fait que celui-ci est plus détaillé et précis, notamment en ce qui concerne les

\footnotetext{
${ }^{49}$ Gautier-Dalché, 2005
} 
instructions pour franchir le chenal du Four et le raz de Sein, passages délicats pour les navigateurs. Les côtes de la Bretagne sont d'ailleurs beaucoup mieux décrites que le littoral aquitain, au point que l'auteur n'a pas jugé bon de créer un chapitre particulier au Poitou ou à la Gascogne, rattachés tous deux à la Bretagne.

«Il y a une chose absolument nécessaire à ceux qui naviguent, et qu'il faut rechercher avec le plus grand soin et la plus grande réflexion : c'est d'avoir avec soi, sur le navire, des guides qui sachent les routes de la mer et les connaissances des terres, et ce qui est à éviter et ce qui ne l'est pas $^{50}$. »Cette dernière phrase du Liber Nautarum introduit en quelque sorte la matière du De viis maris qui le suit dans les manuscrits et qui mentionne aussi la nécessité de posséder des guides, par exemple devant Welle (la rivière Welland, en Angleterre) « où il y a des sables dangereux et il n'y a pas de passage sûr, excepté quand la mer est pleine et quand on a un guide qui en connaît la profondeur ${ }^{51}$. »

Les navires croisés allemands, en 1189, durent eux aussi recourir aux connaissances de guides pour les conduire de Belle-île - voire même de Saint-Mathieu - à La Rochelle par « les détours sinueux de la mer ». Le contexte du récit donne à penser que ces guides étaient des Bretons, et c'est peut-être d'eux que l'auteur a appris le nom breton de Belle-île et ce qu'il sait sur l'emploi de la langue bretonne. Al-Idrîsi note pour sa part que certains, doués des connaissances et de l'audace nécessaires, osent s'hasarder sur les îles inhabitées autour de la Bretagne et pratiquent le cabotage sans s'éloigner de la terre ${ }^{52}$.

Les habitants du littoral breton avaient appris à en déjouer les pièges, et les seigneurs locaux n'ont pas tarder à monnayer cette compétence, comme en témoigne les coutumes des vicomtes de Léon à Saint-Mathieu, et notamment le sceau de conduite qui, une fois acquitté, permettait aux navires franchissant les raz de s'attacher les services d'un guide local ${ }^{53}$. Et $c^{\prime}$ est quelques mois avant le début de son règne que les navires de croisés germaniques vont utiliser les services de pilotes bretons pour rallier La Rochelle depuis la pointe Saint-Mathieu et Belle-île Il est possible d'imaginer qu'ils aient fait appel à des lamaneurs léonards après avoir acquitté un sceau de conduite auprès du receveur du vicomte de Léon, dans le port de Saint-Mathieu.

La nécessité de sécuriser les routes commerciales est donc une raison supplémentaire à la rédaction des routiers atlantiques, que l'on peut vérifier en comparant l'article 13 des Rôles d'Oléron avec le De viis maris : cet article présente en effet certaines similitudes avec le routier atlantique qui a servi de source au De viis maris de Roger de Howden, si ce n'est que l'itinéraire est orienté cette fois-ci du Sud vers le Nord. On y rencontre à nouveau La Rochelle, Batz et Ouessant, ainsi que Yarmouth, noté Geruenne dans le De viis maris ${ }^{54}$.

\footnotetext{
50 « Estque notandum quod ualde necessarium est tranfretantibus et cum omni diligentia et studio perquirendum ut secum in naui conductores habeant qui uias maris et cognitiones terrarum, et que uitanda sunt et que non uitenda cognoscant. », Liber Nautarum, éd. Gautier-Dalché, 2005, p. 172, traduction française p. 83.

51 «Deinde est Welle, ubi sunt arene multe periculose, et non est ibi securum transitus, nisi cum mare plenum fuerit, et nisi habeatur conductor qui profunditatem illius sciat,... », De viis maris, éd. Gautier-Dalché, 2005, p. 176. ${ }^{52}$ Al-Idrîsî, VI,1, 420-421.

${ }^{53}$ Voir supra.

${ }^{54}$ Gautier-Dalché, 2005, p. 177. Il s'agit de l'actuel Great Yarmouth dans le comté de Norfolk, et non du port de l'île de Wright.
} 


\section{4 - Conclusion}

En 1224, parmi les bourgeois de La Rochelle ayant prêté serment au roi de France, les Bretons représentaient environ un tiers des personnes originaires d'un lieu distant de 100 Kms ou plus du port de l'Aunis. En tout état de cause, ils formaient le groupe ethnique le plus nombreux. Il est probable que le port de La Rochelle ait attiré des émigrés bretons dès sa création en 1137, mais leur présence est dans la ville portuaire même est indiscernable avant la toute fin du $12^{\text {ème }}$ siècle, alors qu'on les trouve en Charente et en Saintonge dès le $11^{\text {ème }}$ siècle, voire bien plus tôt ${ }^{55}$.

Il semble que la courbe de l'émigration bretonne vers La Rochelle ait suivi le développement économique de la cité, qui connaît une évolution rapide à partir du dernier quart du $12^{\text {ème }}$ siècle. C'est aussi dans ces dernières décennies du $12^{\text {ème }}$ siècle que l'on peut placer la création des brefs de mer et la rédaction primitive des Rôles d'Oléron, qui portent témoignage de la part prise par la Bretagne ducale et par les ports du Golfe de Gascogne - en particulier La Rochelle - dans la mise en place d'un cadre juridique permettant de réglementer le commerce maritime.

Enfin, les routes maritimes empruntées par les croisés et les pèlerins dans ces mêmes années décrivent un itinéraire de cabotage entre la pointe Saint-Mathieu et La Rochelle, connu et pratiqué par des pilotes bretons maîtrisant parfaitement la navigation côtière.

\section{Bibliographie}

Aimeri Picaud. Le Guide du pèlerin de Saint-Jacques de Compostelle. Traduction Michel Record, éditeur scientifique: Fidel Fita. Bordeaux, Toulouse: éd. Sud-Ouest, Association de coopération interrégionale Les Chemins de Compostelle, 2006.

Al Idrîsî, Muhammad. La première géographie de l'Occident. Traduction du chevalier Jaubert revue par Annliese Nef. Présentation et notes par Henri Bresc et Annlise Nef. Paris: Flammarion, 1999.

Bémont, Charles, 1919. «Le coutumier de l'île d'Oléron.», Bulletin philologique et historique, 246-340.

Boissonnade, Prosper, 1924. «La renaissance et l'essor de la vie maritime en Poitou, Aunis et Saintonge du 10 ème au 15 ${ }^{\text {ème }}$ siècle. ", Revue d'histoire économique et sociale, 12, 259-325.

Boissonnade, Prosper, 1926. «La navigation intérieure du Poitou et des Charentes, leur place dans l'histoire de la navigation fluviale de la France du $9^{\text {ème }}$ au $20^{\text {ème }}$ siècle. », Bulletin de la Section des sciences économiques et sociales du CTHS, 107-124.

Boivin, Jeanne-Marie, 1993. L'Irlande au Moyen-Âge : Giraud de Barri et la Topographia Hibernica (1188). Paris : Champion.

Boutoulle, Frédéric, 2000. «La vigne et le négoce du vin en Bordelais et Bazadais (fin 11 début 13ème siècle). », Annales du Midi, 112, 275-298.

Bruhat, L., 1901. La seigneurie de Châtelaillon. La Rochelle : Texier.

\footnotetext{
55 Voir notre article sur les Bretons en Saintonge : Marquand, 2010, 145-167.
} 
Cassard, Jean-Christophe, 1998. Les bretons et la mer au Moyen-Âge. Des origines au milieu du 14 ème siècle. Rennes : PUR.

Chédeville, André, 1974. «L'immigration bretonne dans le royaume de France du 11 ème au début du $14{ }^{\text {ème }}$ siècle.», $A B P O, 81,301-343$.

Chédeville, André, 1992. "L'anthroponymie bretonne. ", Genèse médiévale de l'anthroponymie moderne. Tours : Publication de l'Université de Tours, tome II-1, 9-40.

Darsel, Joaquim, 1966. «Contribution de la Bretagne à l'élaboration d'un droit de mer. », Bulletin philologique et historique, 1, 1-14.

Delafosse, Marcel, 1955. "Noms de famille rochelais en 1224.", Recueil de travaux offerts à Clovis Brunel par ses amis, collègues et élèves. Paris: Société de l'Ecole des Chartes, I, 339344.

Deniel, Michel, 1971. Etude d'histoire maritime : les rôles d'Oléron du Moyen-Age au 17ème siècle. Mémoire de maitrise d'histoire, UBO.

Dion, Roger, 1959. Histoire de la vigne et du vin en France des origines au 19ème siècle. Paris: Clavreuil.

Favreau, Robert, 1987. « Les débuts de la ville de la Rochelle. », CCM, 30, 3-32.

Favreau, Robert, 1990. «Les débuts de l'histoire de l'Aunis. », BSAO, 3, 11-38.

Garaud, Marcel, 1964. Les châtelains du Poitou et l'avènement du régime féodal, 11 $1^{\text {ème }}$ et $12^{\text {ème }}$ siècles. Poitiers : Société des antiquaires de l'Ouest.

Gautier-Dalché, Patrick, 2005. Du Yorkshire à l'Inde : une 'géographie' urbaine et maritime de la fin du 12ème siècle (Roger de Howden ?). Genève : Droz.

Guillaume le Breton. La Philippide. Traduction de François Guizot, Collection des mémoires relatifs à l'histoire de France, Paris : Brière, 1825.

Hardy, Thoma Duffus, 1835. Rotuli litterarum patentium in turri Londinensi asservati. Londres, vol. I, pars 1.

Henri d'Andeli. « La Bataille des Vins. », Les Dits d'Henri d'Andeli, textes édités, traduits et présentés par Alain Corbellari. Paris : Champion, 2003, 2 vol.

Jeanne-Rose, Olivier, 2001. «Une description peu connue du littoral poitevin à la fin du $12^{\text {ème }}$ siècle. », BSAO, 15, 199-210.

Lachiver, Marcel, 1988. Vins, vignes et vignerons : histoire du vignoble français. Paris : Fayard.

«Le serment de fidélité des habitants de la Rochelle au roi de France en 1224. », Archives historiques $d u$ Poitou, 20, 1889, 232-261. Téléchargeable sur le site Gallica: http://gallica.bnf.fr/ark:/12148/bpt6k209487h/f242.image.r=archives+historiques+poitou+2 0.langFR

Marquand, Patrice, 2010. «Les relations culturelles entre Bretagne et Saintonge au Moyen Âge et la mention de Perceval dans un poème du troubadour Rigaut de Barbezieux. », Cultures, langues et imaginaires de l'arc atlantique. Actes du colloque international de Rennes, 6-8 septembre 2007. Rennes : TIR, 145-167.

Morice, Pierre-Hyacinthe, Dom, 1742-46. Mémoires pour servir de preuves à l'histoire ecclésiastique et civile de Bretagne. Paris : Osmont.

Narratio de itinere navali peregrinorum Hieroslymam tendentium et Silviam capentium. Edited by Charles Wendell David, Proceedings of the American Philosophical Society, t. 81, 1939, pp. 591-676.

Pardessus, Jean-Marie, 1828. Collection de lois maritimes antérieures au $18^{\mathrm{e} m e}$ siècle. Paris : Imprimerie Royale.

Plaine, Dom François, 1881. Vie de Saint-Maurice (de Loudéac), abbé de Langonnet et de Carnoët (1113-1191). Quimperlé : Clairet. 
Planiol, Marcel, 1896. La très ancienne coutume de Bretagne avec les assises, constitution de Parlement et ordonnances ducales suivies d'un recueil de textes divers antérieurs à 1491 . Rennes : Plihon et Hervé.

Planiol, Marcel, 1935-55[1981-84]. Histoire des institutions de la Bretagne. Mayenne: Association pour la publication du manuscrit de M. Planiol, 5 vol.

Pocquet du Haut Jussé, Barthélémy-Amédée, 1946. «Le plus ancien rôle des comptes du duché, 1262. ", MSHAB, 26, 49-68.

Pocquet du Haut Jussé, Barthélémy-Amédée, 1959. "L'origine des brefs de sauveté. », $A B P O$, 46, 255-262.

Renouard, Yves, 1952. "Le grand commerce des vins au Moyen Âge. », Revue historique de Bordeaux et du département de la Gironde, p. 5-18, repris dans Etudes d'histoire médiévale, Paris : SEVPEN, 1968, tome 1, 235-248.

Renouard, Yves, 1959. «Le grand commerce des vins de Gascogne au Moyen Âge. », Revue historique, 221, 267-304, repris dans Etudes d'histoire médiévale, Paris : SEVPEN, 1968, tome 1, 297-335.

Renouard, Yves, 1961. "Le rayonnement de La Rochelle en Occident à l'aube du 13ème siècle.», Bulletin philologique et historique, 79-94, repris dans Etudes d'histoire médiévale, Paris : SEVPEN, tome 2, 1019-1033.

Saint-Marc, C. de, 1909. « Notes pour servir à l'histoire du canton de Frontenay-RohanRohan. », Mémoires de la société scientifique et historique des Deux-Sèvres, 5, 329-358.

Shephard, James, 1983. Les origines des Rôles d'Oléron. Mémoire de maittrise en histoire médiévale, Université de Poitiers.

Shephard, James, 1985. Les Rôles d'Oléron. Etude des manuscrits et édition du texte. Mémoire de DEA en histoire médiévale, Université de Poitiers.

Tonnerre, Noël-Yves, 1998. "Quelques remarques sur les origines de Machecoul. », Mondes de l'Ouest et villes du monde. Regards sur les sociétés médiévales. Mélanges offerts en l'honneur d'André Chédeville. Rennes : PUR, Société d'histoire et d'archéologie de Bretagne, 155-164.

Touchard, Henri, 1956. "Les brefs de Bretagne. », Revue d'histoire économique et sociale, 34, 116-140.

Williston, James H., 1992. Le Coutumier d'Oléron. Edition et traduction annotées. Poitiers : Société des Antiquaires de l'Ouest.

\section{Abréviations}

$\begin{array}{ll}\text { ABPO } & \text { Annales de Bretagne et des Pays de l'Ouest } \\ \text { BSAO } & \text { Bulletins de la Société des Antiquaires de l'Ouest } \\ \text { CCM } & \text { Cahiers de Civilisation Médiévale } \\ \text { CTHS } & \text { Comité des Travaux Historiques et Scientifiques } \\ \text { MSHAB } & \text { Mémoires de la Société Historique et Archéologique de Bretagne } \\ \text { PUR } & \text { Presses Universitaires de Rennes } \\ \text { UBO } & \text { Université de Bretagne occidentale } \\ \text { SEVPEN } & \text { Service d'Edition et de Vente des Publications de l'Education Nationale }\end{array}$

